\title{
A decision support model for multi-attribute group decision making using a multi-objective optimization approach
}

\author{
Jian Xiong ${ }^{\text {a, } b *}$, Yingwu Chen ${ }^{\text {a }}$, Kewei Yang a, c , Jing Liu ${ }^{\text {d,b }}$ \\ ${ }^{a}$ Department of Management, College of Information System and Management, National University of Defense \\ Technology Changsha, 410073, Hunan, P.R.China \\ E-mail: xiongjian1984@hotmail.com \\ ${ }^{b}$ School of Engineering and Information Technology,University of New South Wales at the Australian Defence \\ Force Academy, Canberra ACT, 2600, Australia \\ ${ }^{c}$ Department of Computer Science, University of York, York, YO10 5GH, U.K. \\ ${ }^{d}$ Key Lab of Intelligent Perception and Image Understanding of Ministry of Education of China, Xidian \\ University, 710071, China \\ Received 20 July 2011 \\ Accepted 20 August 2012
}

\begin{abstract}
The multi-attribute group decision making (MAGDM) problem has received considerable attention in the decision analysis field and fruitful achievements have been reported in the literature. This paper focuses on the MAGDM in which the subjective absolute judgement on alternatives with respect to evaluating attributes are represented by fuzzy numbers. This paper employs the consensus degree to measure the agreement level of a MAGDM solution and develops a new measure degree-departure degree to evaluate how far the decision makers from their initial decision preferences. Based on these two conflicting measure degrees, the decision process of MAGDM is modelled as a multi-objective optimization problem. A decision support model (DSM) for MAGDM is proposed. The proposed DSM, incorporating five implementing phases, aims at obtaining acceptable decision solution(s) by solving the multi-objective optimization problem and conducting an interactive procedure with decision makers. In case study, this paper takes the alternative selection problem about hydroelectric project to illustrate the phases and procedure of the proposed DSM.
\end{abstract}

Keywords: multi-attribute group decision making, multi-objective optimization, consensus degree, departure degree, decision support model.

\section{Introduction}

With the increase of complexity, most decision making problems involve multiple decision maker$\mathrm{s}$ (DMs) and multiple evaluating attributes. Such kind of problems are referred to as Multi-Attribute Group Decision Making (MAGDM) problems.The
MAGDM has been extensively studied and applied in various areas and practices s $^{1,2,3,4,5,6}$. Despite diversity of decision approaches used, the MAGDM mainly focuses on two aspects:(1) evaluating alternatives with respect to the multiple attributes and (2) obtaining the group (collective) decision result.

The evaluation process for the attribute weight-

\footnotetext{
${ }^{*}$ Corresponding author, e-mail: xiongjian1984@hotmail.com (J.Xiong).
} 
$s$ and for the performance ratings of the alternatives on attributes may involve two types of judgemen$\mathrm{t}$ : comparative judgement and absolute judgemen$t^{7,8}$. In decision process, comparative judgements may be used to compare the relative importance of evaluating attributes and to compare the performance of each alternative with that of other alternatives on each evaluating attribute with relative measuremen$\mathrm{t}$, while absolute judgements are commonly used to rate the alternatives individually with respect to each evaluating attribute with an absolute measurement scale ${ }^{8}$. Because of the limitation on knowledge, skill and experience related to the problem domain, it is difficult for the DMs to precisely evaluate the alternatives with respect to all evaluating attributes. Instead, DMs may express their preferences by imprecise formats, such as value ranges, linguistic variables and fuzzy numbers ${ }^{9}$. Considerable efforts have been attached to the MAGDM with inaccurate or incomplete information and many approaches to solve this problem have been reported in the existing literature $11,10,5,12,13,14,15,16,17,18,19$.

In MAGDM, DMs usually come from different specialty fields, thus have different knowledge structures and levels, experiences and personalities. As a result, DMs often have diverse opinions. In such a case, it is important to obtain a group satisfactory solution which is the most acceptable by the group of individuals as a whole ${ }^{6}$. In order to obtain the most satisfactory group solution, consensus of the group decision solution is employed to identify the agreement level amongst all DMs. Consensus is defined as a state of mutual agreement among members of a group where all opinions have been heard and addressed to the satisfaction of the group ${ }^{10}$. A consensus reaching process is a dynamic and iterative process composed by several rounds where the experts express, discuss, and modify their preferences ${ }^{19}$. How to obtain the maximum consensus for the given problem is a hot issue which has been received considerable attention in recent years, and various approaches and methods have been developed to achieve this aim $11,13,20,17,21,22,19,23$.

However, in practical group decision making, pursuing of maximum consensus degree of group solution may be not the whole story. In some kind- s of group decision making problems, DMs usually stand for different stakeholders. The solution of MAGDM is often achieved by compromises and negotiations through an interactive and iterative procedure $^{24}$, and this process is implemented by changing the DMs' opinions and preferences according to the decision result in each round until the stop criteria are achieved ${ }^{17,19}$. Thus, the final decision solution may depart from the most preferable one for each D$\mathrm{M}$. The $\mathrm{DM}(\mathrm{s})$ losing in the negotiation process may be influential enough to sabotage the compromise solution and prevent its implementation ${ }^{25}$. This case is especially true for the decision making on considerably complex projects. For example, in the alternative selection of a huge hydroelectric project, the DMs may include stakeholders who stand for the perspectives of economy, environment, society, military, political and so on. The stakeholder(s) losing in the compromise process may refuse to accept the selected alternative and then prevent its execution or request to develop new alternatives. A real case to illustrate this situation is that the indigenous group$s$ in Brazil's Amazon rain forest were trying to halt the construction of a huge hydroelectric project ${ }^{26}$.

Thus, a satisfactory solution of a MAGDM should not only have a great consensus degree, but also keep the DMs' initial preference as much as possible. To do that, this paper employs two measurements for the collective decision solution for the MAGDM: consensus degree and departure degree. The former is used to measure the agreement level of the collective decision solution amongst al1 DMs and the latter reflects how far the individual DM departs from his/her most preferable value. It is clear that consensus degree and departure degree are conflicting to a certain extend. So the decision process of MAGDM itself is a multi-objective optimization problem which aims at pursuing the decision solutions with a higher consensus degree and a lower departure degree. This paper develops a decision support model (DSM) for the MAGDM which incorporates the multi-objective optimization process to obtain the Pareto-optimal group decision solutions with simultaneous consideration of the two degree measurements. Additionally, the proposed DSM employs the concept of proximity measures to 
evaluate the distances between the DMs' individual preferences and the group one ${ }^{13,17,19}$. The proximity measures can be used to generate the advices about which $\mathrm{DM}(\mathrm{s})$ need to change their preference values in the iterative compromise process.

The rest of the paper is set out as follows. In Section 2, we describe the MAGDM problem in which the absolute judgement given by DMS is represented as triangular fuzzy number. In Section 3, we present the proposed decision support model for the MAGDM addressed in this paper. The multiobjective optimization model is developed in this section. A case study about selection of alternatives for hydroelectric project is given in Section 4. Finally we drive our conclusions in the last section.

\section{Preliminary}

\subsection{Problem Description}

As aforementioned, the evaluation in MAGDM includes comparative judgement and absolute judgement. In this paper, we restrict our research on absolute judgement in which DMs express their assessments about the performance of alternatives in grades on a numerical scale. With absolute judgement, DMs can rate the performance of an alternative, independent of other alternatives ${ }^{8}$. Due to the complexity of the problem setting and the limitation on DMs' expertise, the evaluation process is intrinsically imprecise.

Linguistic terms have been found intuitively easy to use in expressing the imprecision of the DMs' evaluation $^{27,28,8}$. Fuzzy set theory is employed in existing studies and applications to characterize each linguistic term by a fuzzy number for representing its approximate value ${ }^{13,29,17,19}$. In this research, we assume that the evaluating value of each attribute given by DMs can be represented as a triangular fuzzy number ${ }^{30}$, expressed as a triple $(a, b, c)$ with its membership function defined as

$$
\mu_{A}(x)=\left\{\begin{array}{l}
(x-a) /(b-a), a \leqslant x \leqslant b, \\
(c-x) /(c-b), \quad b \leqslant x \leqslant c, \\
0, \quad \text { otherwise },
\end{array}\right.
$$

where $b$ is the most possible value of the fuzzy num- ber $A, a$ and $c$ are respectively the lower and upper bounds, used to reflect the fuzziness of the subjective evaluation.

In the fuzzy linguistic group decision making problem, an important issue to analyze is the "granularity of uncertainty", i.e., the cardinality of the linguistic term set ${ }^{19}$. The granularity of linguistic term set should be small enough so as not to impose useless precision levels on the users but large enough to allow a discrimination of the assessments in a limited number of degrees ${ }^{19}$. So we identify the 1-9 ratio scale as the standard numerical scale of evaluating value. For example, in the evaluation of a hydroelectric project alternatives with respect to the attribute "the importance on prevention of flood": $1=$ not important, $3=$ not very important, $5=$ important, $7=$ very important, $9=$ extremely important. The $1-9$ ratio scale has been proven to be an effective measurement scale for reflecting the qualitative information of a decision problem ${ }^{31}$. Thus, in Eq.(1), $a, b$ and $c$ are defined in the interval $[0,10]$, and $a \leqslant b \leqslant c$. Then, the MAGDM with fuzzy absolute judgement can be described as follows.

There is a finite set of alternatives, $X=$ $\left\{x_{1}, x_{2}, \cdots, x_{n}\right\}(n \geqslant 2)$, and a group of DMs (experts) who stand for the different stakeholders of the decision problem, $E=\left\{e_{1}, e_{2}, \cdots, e_{m}\right\}(m \geqslant 2)$. Each $\mathrm{DM} e_{k}$ provides his/her evaluation on $X$ with respect to a set of attributes $C=\left\{c_{1}, c_{2}, \cdots, c_{q}\right\}(q \geqslant 2)$. The weight vectors $\omega=\left\{\omega_{1}, \omega_{2}, \cdots, \omega_{m}\right\}$ and $\lambda=$ $\left\{\lambda_{1}, \lambda_{2}, \cdots, \lambda_{q}\right\}$ respectively represent the weight$\mathrm{s}$ of DMs and evaluation attributes, and satisfying $\sum_{i=1}^{m} \omega_{i}=1\left(\omega_{i} \geqslant 0\right)$ and $\sum_{j=1}^{q} \lambda_{j}=1\left(\lambda_{j} \geqslant 0\right)$.

Let $A_{k}=\left(a_{i j}^{k}\right)_{n \times q}$ be a numerical decision matrix, where $a_{i j}^{k}$ is an evaluation value given by the DM $e_{k} \in E$ for the alternative $x_{i} \in X$ with respect to the attribute $c_{j} \in C$. Evaluation value $a_{i j}^{k}$ is represented as a triangular fuzzy number $\left(a_{i j}^{k_{\text {lower }}}, a_{i j}^{k_{\text {most }}}, a_{i j}^{k_{\text {upper }}}\right)$, where $a_{i j}^{k_{\text {most }}}$ indicates the most preferable value given by a DM, $a_{i j}^{k_{\text {lower }}}$ and $a_{i j}^{k_{\text {upper }}}$ are the lower and upper bounds of the evaluation value, which can be accepted by the DM.

The satisfactory solution of group decision making problem is often achieved by an interactive and iterative process. In real implementation, this inter- 
active process is time and resource intensive, i.e., the DMs come from different fields and are geographically distributed, therefore it will take days or week$s$ to hold a round of discussion with all DMs. So it is important to reduce the number of discussion rounds, while obtaining the satisfactory decision solution. To do that, we develop a computer-aided decision support model (DSM) in which a multiobjective optimization process is employed to obtain the more satisfactory decision solution(s). We assume that there is an upper level decision maker who is responsible for processing the decision information collected from all DMs, holding discussion with all DMs, and selecting the final decision if more than one satisfactory decision solution exists. For example, in China, the State Council plays such a role in the construction of a huge hydroelectric project. In the DSM, if the decision solution is not satisfactory, the upper decision maker can adjust the most preferable evaluation value of a DM within the upper and lower bounds. The decision matrices after adjustments are denoted as $\widehat{A}_{k}=\left(\widehat{a}_{i j}^{k}\right)_{n \times q}$, where $\widehat{a}_{i j}^{k}=\left(a_{i j k}^{k_{\text {lower }}}, \hat{a}_{i j k}^{k_{\text {most }}}, a_{i j k}^{k_{\text {upper }}}\right)$. If there is still not any satisfactory decision solution after the adjustmen$\mathrm{t}$ process, this dilemma as well as advices will be reported to DMs and they will be suggested change their evaluation values. When DMs accept the advices and change their evaluation values, the decision information is recollected and decision process is continued until the stopping criteria are reached.

\section{Decision Support Model for MAGDM}

Real important decisions are often difficult to make. To alleviate such difficulty it would be necessary and desirable to use some kind of decision support ${ }^{17}$. The aim of this paper is to develop a decision support model (DSM) for MAGDM based on a multi-objective optimization process where two types of measurements-consensus degree and departure degree are taken as the optimization objectives. The framework of the proposed DSM for MAGDM is presented in Figure 1. The DSM begin$\mathrm{s}$ with collecting and processing decision information. Based on the input information, consensus and departure degrees will be calculated. If it exists so- lution which satisfies the preset threshold values of the two degrees, the final selection process is implemented to identify the final solution(s). Otherwise, the multi-optimization process will be activated to search other solutions which are expected to satisfy the threshold conditions. If there is still no such satisfied solution, an interactive procedure with DM$\mathrm{s}$ will be carried out to guide some DMs to adjust their decision preferences. The whole procedure is repeated till satisfied solutions are obtained. Specifically, the DSM for MAGDM develops its activity in five phases as follows.

1) Preliminary process of decision information. In this phase, the decision matrices are processed. Firstly, in order to facilitate the comparison of all elements in the decision matrices, the evaluation values about cost attributes are converted to the values about benefit attributes. Then the decision matrices are normalized if necessary.

2) Computation of consensus degree and departure degree. In this phase, consensus degree and departure degree are computed. To do that, a similarity measure is defined to calculate the coincidence amongst DMs' opinions or preferences and the membership function of triangular fuzzy number is used to measure the coincidence between one decision solution and the DMs' initial decision preferences. The threshold values of consensus degree and departure degree are predefined, denoted as $\eta$ and $\gamma$ respectively. If the consensus degree is lower than threshold or the departure degree is greater than threshold, the decision solution is considered as unacceptable. It is clear that in the first round computation, the departure degree is equal to 0 . However, we are aware of that presetting appropriate threshold values is not an easy task. The threshold values will depend on the problem domain. When the consequences of the decision to be made are of utmost importance, the threshold of consensus should be set as high as possible, usually greater than 0.8 . On the contrary, in cases where the consequences are not so serious, it is reasonable to set the threshold value of consensus as close as $0.5^{19}$. Similarly, the threshold value of departure degree should be set as low as possible if the consequences are very important. In our research, we assume that the decisions are 


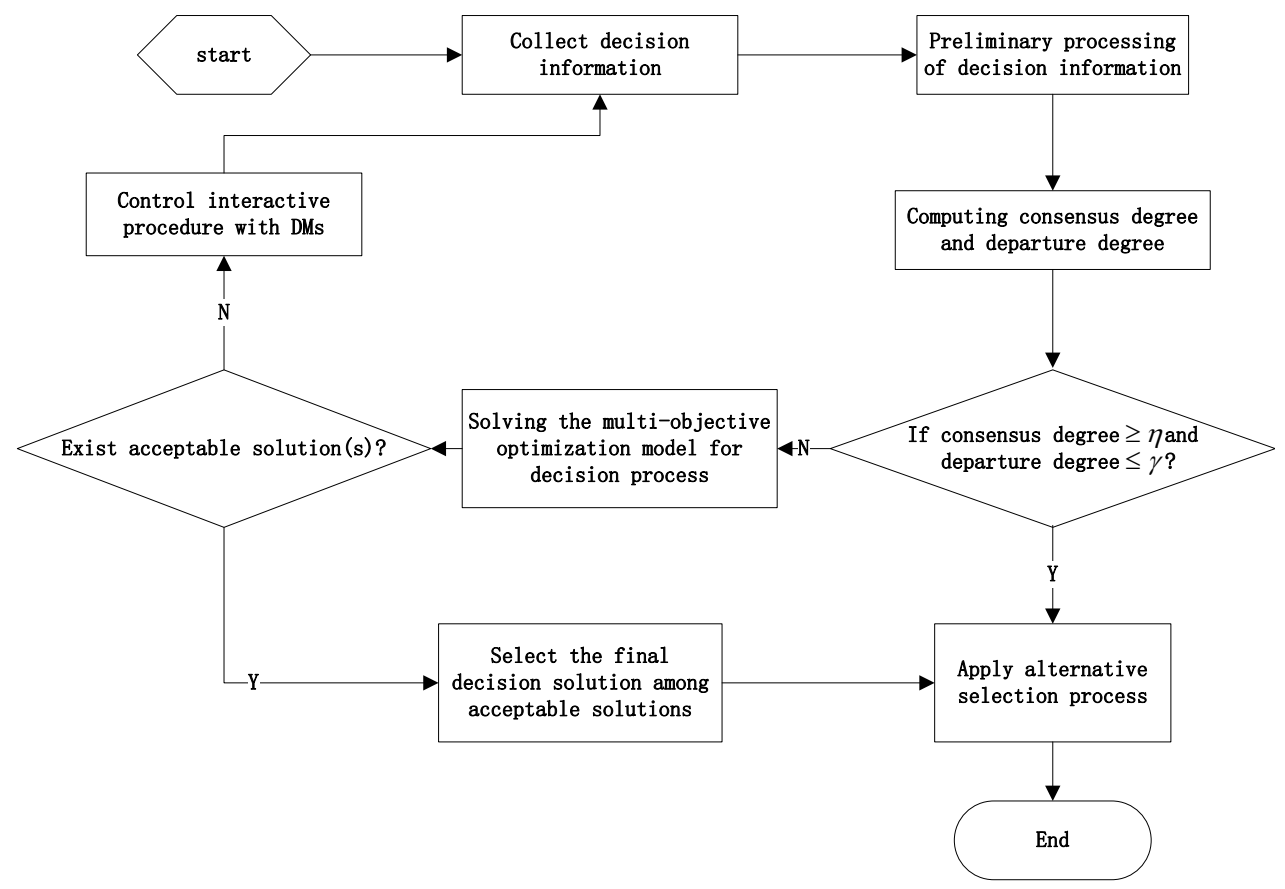

Figure 1: Framework of the proposed decision support model for MAGDM

serious and of high importance. For the sake of simplicity in case study, we respectively set the values of $\eta$ and $\gamma$ as 0.85 and 0.15 .

3) Obtaining the Pareto optimal decision solutions via a multi-objective optimization process. If the two measure degrees of decision solutions satisfy the predefined acceptance conditions, then the DSM goes to phase 5) to apply the alternative selection process. Otherwise, a multi-objective optimization process is employed to obtain the satisfactory decision solutions. In some cases, there is no any decision solution satisfying the predefined acceptance conditions after the optimization process. Then, the DSM goes to phase 4) to conduct a interactive procedure with DMs.

4) Controling the interactive procedure with DMs. In this phase, a discussion will be held among all or some of the DMs. The decision solutions as well as the advice about how to change the evaluation values should be reported to DMs who will be suggested to modify their initial decision preferences or opinions. In this feedback process, the DSM will identify the DM(s) who need to change their decision preferences in the next round according to the proximity. After that, the DSM repeats the process from the first phase. In real problems, some DMs may not accept the advices and refuse to change their decision preferences. When such a dilemma occurs, it is necessary to develop new alternatives. This situation is out of the scope of this research, we assume that DMs will accept the advices generated by the DSM. In order to avoid that the interactive procedure dose not converge after many rounds of discussion, we employ a maximum number of rounds to be developed in the interactive procedure, denoted as Maxcycles ${ }^{32,16,17}$.

5) Applying the alternative selection process. If the decision solution satisfies the predefined acceptance conditions, then selection process is applied to calculate the performances of alternatives and select the preferable one(s). What should be noted is that there may be more than one decision solution which satisfies the predefined acceptance conditions. In such situation, as all decision solutions are feasible, the identification of decision solution is up to the trade-off between consensus degree and depar- 
ture degree. We concentrate our research on the entire structure of the DSM and the trade-off approach is beyond the scope of this paper.

\subsection{Preliminary process of decision information}

In general, there are benefit attributes and cost attributes in MAGDM ${ }^{23}$. In order to facilitate interattribute comparisons and to measure all attributes evaluation values in dimensionless units, in this phase we transform the evaluation values for cost attributes to that for benefit attributes, and then normalize the evaluation value to the interval $[0,1]$. For cost attributes, the transformed evaluation value is denoted as $a_{i j}^{\prime k}$, given as follows:

$$
a_{i j}^{\prime k}=\left(a_{\max }-a_{i j}^{k_{\text {upper }}}, a_{\max }-a_{i j}^{k_{\text {most }}}, a_{\max }-a_{i j}^{k_{\text {lower }}}\right)
$$

where $a_{\max }$ is the maximum value of the standard numerical evaluation scale. As this paper employs the 1-9 ratio scale as the numerical scale of evaluation value, we set $a_{\max }$ to 10 . Then, we normalize the evaluation value into the interval $[0,1]$. For each $\mathrm{DM} e_{k} \in E$, the normalized decision matrix is represented as $\widetilde{A_{k}}=\left(\widetilde{a}_{i j}^{k}\right)_{n \times q}$, where

$$
\widetilde{a}_{i j}^{k}=\left(\frac{a_{i j}^{k_{\text {lower }}}}{a_{\max }}, \frac{a_{i j}^{k_{\operatorname{most}}}}{a_{\max }}, \frac{a_{i j}^{k_{u} \text { pper }}}{a_{\max }}\right)
$$

\subsection{Computation of consensus degree and departure degree}

Consensus degree is used to measure the level of agreement amongst all DMs. The computation of consensus degree requires the use of some similarity or coincidence functions to obtain the level of agreement ${ }^{13,14,17}$. These similarity or coincidence functions can be defined using the traditional distance measurement, e.g., Euclidean distance. As in this research the evaluation value is given by triangular fuzzy number, we define the measure of consensus degree based on the concept of distance between triangular fuzzy numbers. According to the existing literature ${ }^{33,34}$, there are many distance measurement functions for fuzzy numbers. To compare the fuzzy evaluation value, we use the concept of vertex method ${ }^{35}$ to calculate the distance between fuzzy numbers. Let $a=\left(a_{1}, a_{2}, a_{3}\right)$ and $b=\left(b_{1}, b_{2}, b_{3}\right)$ be two triangular fuzzy numbers, the distance between which is given by

$d^{\prime}(a, b)=\sqrt{\frac{1}{3}\left[\left(a_{1}-b_{1}\right)^{2}+\left(a_{2}-b_{2}\right)^{2}+\left(a_{3}-b_{3}\right)^{2}\right]}$

In the problem addressed in this research, the value $a_{2}$ and $b_{2}$ represent the most preferable evaluation value given by DMs. Thus, these values indicate the best level of a DM's knowledge and expertise towards to an alternative with respect to a given evaluating attribute. So the most preferable value plays a more important role in comparison of two evaluation values. Then, we modify the vertex method for distance measurement as follows.

$$
\begin{aligned}
& d(a, b)= \\
& \sqrt{\left.\frac{\alpha}{2}\left(a_{1}-b_{1}\right)^{2}+(1-\alpha)\left(a_{2}-b_{2}\right)^{2}+\frac{\alpha}{2}\left(a_{3}-b_{3}\right)^{2}\right]}
\end{aligned}
$$

where $\alpha$ is a constant coefficient in the interval $[0,1]$. Since $\alpha$ indicates the importance of the lower and upper bounds of the fuzzy evaluating values, we suggest the value of $\alpha$ is set to lower than 0.5. In this research $\alpha$ is set to 0.1 with no other specific reason. From the definition of distance in Eq.(5) we can see that if $a$ and $b$ are real numbers, them the distance $d(a, b)$ is identical to the Euclidean distance. Thus, we define a similarity function $s$ between these two triangular fuzzy numbers as follows:

$$
s(a, b)=1-\frac{d(a, b)}{g}
$$

In order to normalize the similarity measurement in the interval $[0,1], g$ is taken the value of maximum numerical scale $a_{\max }$. And note that if the fuzzy evaluation value has been normalized as Eq. (3), then $g$ is set to be 1 . The closer $s(a, b)$ is to 1 the more similar $a$ and $b$ are, while the closer $s(a, b)$ is to 0 the more distant $a$ and $b$ are.

For two DMs $e_{k_{1}}$ and $e_{k_{2}} \in E$, their similarity on an evaluating attribute $c_{j} \in C$ for an alternative $x_{i} \in X$ is given by

$$
s c_{i j}^{k_{1} k_{2}}=s\left(a_{i j}^{k_{1}}, a_{i j}^{k_{2}}\right), \quad i=1, \cdots, n \text { and } j=1, \cdots, q
$$


The similarity on the alternative is obtained by aggregating the similarities on attributes using the additive weight aggregation (AWA) operator, given by

$$
s a_{i}^{k_{1} k_{2}}=\sum_{j=1}^{q} \lambda_{j} s c_{i j}^{k_{1} k_{2}}, \quad i=1, \cdots, n
$$

The consensus degree between two DMs in a decision solution, denoted as $c s^{k_{1} k_{2}}$, is defined as the arithmetic mean of the similarity on alternatives, given by

$$
c s^{k_{1} k_{2}}=\frac{\sum_{i=1}^{n} s a_{i}^{k_{1} k_{2}}}{n}
$$

The consensus degree of a decision solution, called $c s$, is defined as the minimum of consensus degrees for all pairs of DMs in a decision solution, given by

$$
c s=\operatorname{Min}\left(c s^{k_{1} k_{2}}\right), k_{1}, k_{2}=1, \cdots, m \wedge k_{1} \neq k_{2}
$$

If $c s<\eta$, it indicates that the agreement level amongst DMs is lower than the threshold. Then, the data processor can adjust the most preferable evaluation value of DMs in the permitted interval.

Departure degree is used to measure how far the adjusted decision solution from the initial decision solution. In a decision solution after adjustment, for a DM $e_{k} \in E$, the distance between the initial preference value and adjusted preference value for an alternative $x_{i} \in X$ with respect to an evaluating attribute $c_{j} \in C$, denoted as $d_{i j}^{k}$, is defined as follows:

$$
d_{i j}^{k}=1-\mu_{a_{i j}^{k}}\left(\widehat{a}_{i j}^{k_{m o s t}}\right)
$$

where $\mu$ is the membership function of a triangular fuzzy number given by Eq.(1). It is clear that $d_{i j}^{k}$ is in the interval of $[0,1]$ and the closer $d_{i j}^{k}$ is to 0 the closer the $\mathrm{DM} e_{k}$ is to his/her most preferable value on the attribute, the closer $d_{i j}^{k}$ is to 1 the more distant the $\mathrm{DM} e_{k}$ is from his/her most preferable value.

The distance between the initial preference value and adjusted preference value for an alternative $x_{i} \in X$, denoted as $d_{i}^{k}$, is calculated by AWA operator, given by

$$
d_{i}^{k}=\sum_{j=1}^{q} \lambda_{j} d_{i j}^{k}
$$

The departure degree of a DM, denoted as $d p^{k}$, is defined as the arithmetic mean of distance for all alternatives, given by

$$
d p^{k}=\frac{\sum_{i=1}^{n} d_{i}^{k}}{n}
$$

The departure degree of a decision solution, called $d p$, is defined as the maximum of departure degrees for all DMs in a decision solution, given by

$$
d p=\operatorname{Max}\left(d p^{k}\right), k=1, \cdots, m
$$

If $d p>\gamma$, the decision solution is considered as unacceptable.

\subsection{Multi-objective model for the MAGDM process}

In the conventional MAGDM problems, great effort$s$ have been attached to the pursuing of maximum consensus degree of the decision solution. We introduce departure degree as another measurement to the decision solution, then the decision process is modeled as a multi-objective optimization process which simultaneously considers the maximization of consensus degree and the minimization of departure degree. The multi-objective optimization model of MAGDM process is presented as $\operatorname{Model}(1)$, given as follows.

$$
\begin{aligned}
\text { obj.: } & \text { Max } f_{1}=c s(I), \text { Min } f_{2}=d p(I) \\
\text { s.t. : } & a_{i j}^{k_{\text {lower }}} \leqslant \widehat{a}_{i j}^{k_{\text {most }}} \leqslant a_{i j}^{k_{\text {upper }}} \\
& i=1, \cdots, n \\
& j=1, \cdots, q \\
& k=1, \cdots, m
\end{aligned}
$$

where $I$ is the decision solution which consists of decision matrices of all DMs.

Given the predefined threshold values of $\eta$ and $\gamma$, for a decision solution $I$, if $c s(I)<\eta$ or $d p(I)>\gamma$, the decision solution is considered as unacceptable. Then, the DSM solves the multi-objective optimization problem proposed in Model(1) to obtain the 
more satisfactory solutions. We define the acceptable decision solution based on the non-dominated concept as follows.

Definition 1. A decision solution $I$ is said to be a non-dominated solution of Model(1), if and only if there does not exist another decision solution $\bar{I}$ for the consensus degree $c s$ and departure degree $d p$, satisfying the following condition: $\operatorname{cs}(\bar{I}) \geqslant$ $c s(I) \wedge d p(\bar{I}) \leqslant d p(I)$, with strict inequality holding for at least one measure degree.

Definition 2. For a MAGDM problem, the decision solution $I^{*}$ is said to be an acceptable solution if $I^{*}$ is a non-dominated solution for Model (1) and satisfying the following condition: $\operatorname{cs}\left(I^{*}\right) \geqslant \eta \wedge d p\left(I^{*}\right) \leqslant$ $\gamma$.

For solving the proposed multi-objective optimization problem, multi-objective evolutionary algorithm (MOEA) is employed in DSM because of its ability to find multiple non-dominated solution$\mathrm{s}$ in a single run ${ }^{36}$. Although a great number of MOEAs have been suggested since about the early 1990's, we use one of the classic MOEAs NSGA-II ${ }^{37}$ - as the optimization algorithm. NSGAII is an elitism algorithm whose main feature is the fast-non-dominated-sort and the crowding-distanceassignment mechanism. The former creates a mating pool by combining the parent and offspring populations and selecting solutions considering fitness and spread. The latter ensures diversity is maintained among non-dominated solutions.

\subsection{Control the interactive procedure with DMs}

In some cases, we cannot obtain any acceptable decision solution after the optimization process. It indicates that the decision preferences are very different amongst the DMs. In such a case, the DSM will identify the DM(s) who need to change their evaluation values and generate changing direction advices. The upper level decision maker will report this situation as well as advices to the identified $\mathrm{DM}(\mathrm{s})$. Then, the new decision information is collected, and the computing and optimization process aforementioned is repeated.

The proximity measure, which reflects the distance between an individual preference and the col- lective one, is used to identify the $\operatorname{DM}(\mathrm{s})$ who need to change their preferences in the next round. Let $A_{c}=\left(a_{i j}\right)_{n \times q}$ be the collective decision matrix, where $a_{i j}$ is also a triangular fuzzy number represented as $\left(a_{i j}^{\text {lower }}, a_{i j}^{\text {most }}, a_{i j}^{\text {upper }}\right)$. $a_{i j}$ is obtained by aggregating all evaluation values given by all DM$\mathrm{s}$ as shown in Eq.(16).

$$
a_{i j}=\Psi\left(a_{i j}^{1}, a_{i j}^{2}, \cdots, a_{i j}^{m}\right)
$$

where $\Psi$ is the AWA operator, i.e.:

$$
\begin{aligned}
& a_{i j}^{\text {lower }}=\sum_{k=1}^{m} \omega_{k} a_{i j}^{k_{\text {lower }}} \\
& a_{i j}^{\text {most }}=\sum_{k=1}^{m} \omega_{k} a_{i j}^{k_{\text {most }}} \\
& a_{i j}^{\text {upper }}=\sum_{k=1}^{m} \omega_{k} a_{i j}^{k_{\text {upper }}}
\end{aligned}
$$

For each DM $e_{k} \in E$, the proximity measure, denoted as $\mathrm{pm}_{k}$, is defined as the consensus degree between the individual DM and the collective one, given by

$$
p m_{k}=c s^{k c}
$$

where the $\mathrm{DM} e_{c}$ represents the collective one whose decision matrix is obtained by Eq.(17)-Eq.(19). The arithmetic mean of proximities is calculated in each round and the DM whose proximity is below the mean value is identified as the one who should change his/her evaluation value in the next round. It can be represented as: For each DM $e_{k} \in E$, if the proximity measure $\mathrm{pm}_{k}<\overline{p m}_{k}=\frac{\sum_{k=1}^{m} p m_{k}}{m}$, then DM $e_{k}$ is suggested to change his/her preference in the next round.

The change direction advices are represented as direction rules ${ }^{19}$ and are generated based on the comparison of individual decision preferences and collective decision preference. Here we use the most possible value of the triangular fuzzy evaluation value to compare individual decision preferences and the group decision preference. Thus, the direction rules can be described as follows.

Rule 1. For the evaluation value of an alternative $x_{i}$ with respect to an attribute $c_{j}$ given by $\mathrm{DM} e_{k}$, if $a_{i j}^{k_{\text {most }}}-a_{i j}^{\text {most }}<0$, then the DM $e_{k}$ need to increase 
the evaluating value associated with the attribute if it is a benefit attribute or decrease the evaluating value associated with the attribute if it is a cost attribute.

Rule 2. For the evaluation value of an alternative $x_{i}$ with respect to an attribute $c_{j}$ given by $\mathrm{DM} e_{k}$, if $a_{i j}^{k_{\text {most }}}-a_{i j}^{\text {most }}>0$, then the DM $e_{k}$ need to decrease the evaluating value associated with the attribute if it is a benefit attribute or increase the evaluating value associated with the attribute if it is a cost attribute.

Rule 3. For the evaluation value of an alternative $x_{i}$ with respect to an attribute $c_{j}$ given by $\mathrm{DM} e_{k}$, if $a_{i j}^{k_{\text {most }}}-a_{i j}^{\text {most }}=0$, then the DM $e_{k}$ dose not need to change the evaluating value associated with the attribute.

\subsection{Applying the alternative selection process}

After the optimization process and/or the interactive procedure with DMs, the acceptable solutions are obtained. Then the final decision solution is identified by upper level decision maker and the alternative selection process is applied to calculate the performance ratings of all alternatives under the identified decision solution. This paper employs a widely used multiple attribute decision method called the technique for order preference by similarity to ideal solution (TOPSIS) ${ }^{38,39}$ as the alternative selection method for MAGDM. The basic concept of TOPSIS is that the most preferred alternative should not only have the shortest distance from the positive ideal solution (or the best possible alternative), but also have the longest distance from the negative ideal solution (or the worst possible alternative). For the specific details of TOPSIS, readers are referred to ${ }^{8}$.

\section{Case Study}

To illustrate the proposed DSM for MAGDM, an example about selection of alternatives for hydroelectric project is taken as a case study in this research. The problem background is described as follows: China decides to build a hydroelectric project in the Yangzte River basin. For sustainable development, the decision about the project should consider many related aspects. Experts from different fields are called to express their decision preference on dam height and electricity generation capacity, whose different combinations compose the alternatives to be evaluated. Table 1 shows the evaluation attributes for an alternative in hydroelectric project decision problem and the weight vector of attributes is denoted as $\lambda=(0.12,0.18,0.20,0.20,0.16,0,14)$. The group of DMs consists of 5 panel of expert(s) which are from different fields and departments, such as Ministry of Water Resource, National Development and Reform Commission, Ministry of Environmental Protection, Ministry of Civil Affairs, National Disaster Reduction Committee and so on. The group of DMs is denoted as $E=\left(e_{1}, e_{2}, e_{3}, e_{4}, e_{5}\right)$, whose weight vector is $\omega=(0.15,0.2,0.22,0.25,0.18)$. There are 4 alternatives to be evaluated, denoted as $X=\left(x_{1}, x_{2}, x_{3}, x_{4}\right)$. The alternatives stand for different settings of the key indices of the hydroelectric project, i.e., dam height and electricity generation capacity in this research. The decision matrices given by DMs are represented in Table 2.

\subsection{Preliminary process and consensus degree computation}

The evaluating attributes $c_{2}, c_{4}$ and $c_{5}$ are cost attributes, so we transformed the evaluation values with respect to these attributes as Eq.(2). The thresholds of consensus degree and departure degree is predefined to be $\eta=0.85$ and $\gamma=0.15$. The consensus degree of initial decision solution is calculated as $c s=0.8050$. Because $c s<0.85$, the adjustment process of the preference values by solving the multi-objective optimization model is applied.

\subsection{Solving multi-objective optimization model for MAGDM}

In the employed multi-objective genetic algorithm, we used a population size of 100 . And crossover and mutation rates were 0.98 and 0.20 respectively. The evolution was terminated after 5000 generations. Figure 2 shows the obtained non-dominated decision solutions after 5000 generations. It is clear that the figure visualizes a set of non-dominated decision solutions well-spread in the objective sapce. This indicates that consensus degree and departure 
Table 1: Evaluating attributes of MAGDM in a case study.

\begin{tabular}{ll}
\hline Attribute & Description \\
\hline$c_{1}$ & $\begin{array}{l}\text { Economic factor, which reflects the economic benefit resulting from the } \\
\text { project. }\end{array}$ \\
$c_{2}$ & $\begin{array}{l}\text { Environment factor, which reflects the negative affect to the ecology by the } \\
\text { project. }\end{array}$ \\
& $\begin{array}{l}\text { Disaster prevention, factor which reflects the capability to control flood disas- } \\
\text { ter and drought disaster by using the hydroelectric project. }\end{array}$ \\
& $\begin{array}{l}\text { Society factor, which reflects the negative impact to society by the project, } \\
c_{4}\end{array}$ \\
& such as immigration, destroy to cultural heritage and so on. \\
$c_{5}$ & $\begin{array}{l}\text { Military factor, which reflects the project's potential risk when it is attacked in } \\
\text { the war time. }\end{array}$ \\
& Agriculture factor, which reflects agricultural benefit acquired from the project. \\
$c_{6}$
\end{tabular}

degree are strongly conflicting with each other. Since the predefined threshold values of consensus degree and departure degree are 0.85 and 0.15 respectively, there is no acceptable decision solution among these obtained non-dominated solutions and the interactive procedure with DMs is activated.

\subsection{Interactive procedure with DMs}

The vector of proximity measures for all DMs was computed as $(0.9381,0.8987,0.9304,0.9369$, $0.8863)$. The average of proximity measures was $\overline{p m}_{k}=0.9181$. Thus, the DMs $e_{2}$ and $e_{5}$ were identified to be suggested to change there evaluation values in the next round. By considering both group opinion and individual opinions, change direction advices could be generated through the direction rules, i.e., Rule 1, Rule 2 and Rule 3. These advices as well as group opinion were reported to $e_{2}$ and $e_{5}$. Then, they could know their gaps between group opinion on each attribute for each alternative and make some changes on their most preferable evaluating values. We suppose that the decision matrices given by $e_{2}$ and $e_{5}$ after the first round of interactive procedure are shown in Table 3 and 4, respectively.

\subsection{Repeating the degrees computation and optimization process}

In the next round, the consensus degree of decision solution came out as $c s=0.8248$ and at this time the departure degree was equal to 0 . Because $c s$ was stil1 lower that the predefined threshold value 0.85 , the optimization process was applied to obtain more satisfactory decision solutions with the aid of computer. The parameter settings were remained the same as those in the first round. Figure 3 shows the the obtained non-dominated decision solutions after 5000 generations in the second round. It is quite clear from the figure that there are acceptable decision solutions which satisfy $c s \geqslant 0.85$ and $d p \leqslant 0.15$ in the well-spread set of non-dominated solutions. For the sake of clarity, we removed the non-acceptable solutions from the non-dominated set and Figure 4 shows the visualization of acceptable decision solutions in the objective space.

\subsection{Applying the alternative selection process}

The upper lever decision maker identifies the final decision solution from the set of acceptable decision solutions according to the preference and by using 
Table 2: Initial decision matrix given by DM1-DM5.

\begin{tabular}{lcccccc}
\hline DM1 & $c_{1}$ & $c_{2}$ & $c_{3}$ & $c_{4}$ & $c_{5}$ & $c_{6}$ \\
\hline$x_{1}$ & $(7.5,7.8,8.2)$ & $(1.5,2.0,2.5)$ & $(7.9,8.4,8.8)$ & $(2.2,2.6,2.9)$ & $(1.4,1.9,2.6)$ & $(8.3,8.8,9.3)$ \\
$x_{2}$ & $(7.8,8.3,8.8)$ & $(1.3,1.7,2.3)$ & $(7.3,8.0,8.5)$ & $(1.8,2.2,2.7)$ & $(1.8,2.3,2.7)$ & $(8.3,8.9,9.4)$ \\
$x_{3}$ & $(7.4,8.1,8.5)$ & $(1.2,1.6,2.0)$ & $(8.1,8.6,9.1)$ & $(1.3,1.8,2.5)$ & $(1.6,2.0,2.7)$ & $(7.4,8.0,8.9)$ \\
$x_{4}$ & $(8.2,8.8,9.2)$ & $(1.8,2.3,3.0)$ & $(8.0,8.6,9.0)$ & $(2.1,2.7,3.1)$ & $(1.3,1.7,2.2)$ & $(7.9,8.4,9.0)$ \\
DM2 & & & & & & \\
\hline$x_{1}$ & $(8.0,8.4,8.9)$ & $(1.6,2.4,2.9)$ & $(7.3,7.6,8.1)$ & $(2.1,2.7,3.2)$ & $(1.4,1.9,2.5)$ & $(7.0,8.0,8.7)$ \\
$x_{2}$ & $(7.1,7.5,8.0)$ & $(1.9,2.6,3.2)$ & $(8.0,8.3,8.8)$ & $(2.3,2.7,3.0)$ & $(1.6,1.9,2.4)$ & $(7.5,7.9,8.3)$ \\
$x_{3}$ & $(6.8,7.2,7.6)$ & $(1.5,1.9,2.5)$ & $(8.4,9.0,9.4)$ & $(1.9,2.6,3.1)$ & $(2.2,2.7,3.3)$ & $(7.0,7.4,8.0)$ \\
$x_{4}$ & $(7.3,8.0,8.5)$ & $(2.1,2.7,3.2)$ & $(7.1,7.4,8.0)$ & $(1.8,2.3,2.9)$ & $(1.7,2.3,2.9)$ & $(7.3,8.0,8.8)$ \\
DM3 & & & & & & \\
\hline$x_{1}$ & $(7.6,8.1,8.8)$ & $(2.0,2.5,2.9)$ & $(7.8,8.2,8.9)$ & $(2.1,2.4,2.8)$ & $(1.7,2.2,2.6)$ & $(8.2,8.5,9.0)$ \\
$x_{2}$ & $(7.4,7.7,8.5)$ & $(1.3,1.8,2.4)$ & $(6.9,7.5,8.3)$ & $(1.3,1.7,2.2)$ & $(1.5,2.1,2.5)$ & $(8.6,9.0,9.4)$ \\
$x_{3}$ & $(7.6,8.0,8.9)$ & $(1.8,2.2,2.6)$ & $(8.1,8.5,9.0)$ & $(1.8,2.4,2.8)$ & $(1.4,1.8,2.4)$ & $(8.0,8.5,8.9)$ \\
$x_{4}$ & $(8.8,9.1,9.3)$ & $(1.9,2.6,3.0)$ & $(8.0,8.4,9.0)$ & $(1.1,1.6,2.0)$ & $(1.6,1.9,2.2)$ & $(7.8,8.3,8.8)$ \\
DM4 & & & & & & \\
\hline$x_{1}$ & $(8.0,8.3,8.9)$ & $(1.4,1.8,2.2)$ & $(7.7,8.1,8.6)$ & $(2.2,2.5,2.9)$ & $(1.4,1.7,2.4)$ & $(7.3,8.3,9.3)$ \\
$x_{2}$ & $(7.8,8.3,8.8)$ & $(1.6,2.4,2.8)$ & $(7.0,7.6,8.1)$ & $(1.6,2.0,2.6)$ & $(2.0,2.5,2.9)$ & $(8.4,8.8,9.5)$ \\
$x_{3}$ & $(7.6,8.1,8.6)$ & $(1.8,2.3,2.7)$ & $(8.1,8.7,9.1)$ & $(1.3,1.8,2.5)$ & $(1.6,2.2,2.7)$ & $(8.0,8.3,8.8)$ \\
$x_{4}$ & $(8.0,8.5,9.0)$ & $(1.2,1.7,2.5)$ & $(8.4,8.9,9.3)$ & $(2.1,2.5,3.1)$ & $(1.3,1.6,2.2)$ & $(7.9,8.5,8.9)$ \\
DM5 & & & & & & \\
\hline$x_{1}$ & $(8.0,8.3,8.8)$ & $(2.4,2.8,3.4)$ & $(7.0,7.6,8.3)$ & $(1.7,2.1,2.9)$ & $(1.8,2.4,2.9)$ & $(7.3,7.8,8.3)$ \\
$x_{2}$ & $(6.8,7.2,8.0)$ & $(1.9,2.5,2.9)$ & $(7.8,8.2,8.8)$ & $(2.3,2.9,3.2)$ & $(2.3,2.8,3.2)$ & $(7.2,7.6,8.1)$ \\
$x_{3}$ & $(7.0,7.8,8.4)$ & $(1.5,1.9,2.4)$ & $(7.0,7.8,9.0)$ & $(1.7,1.9,2.4)$ & $(1.8,2.3,2.7)$ & $(8.1,8.4,9.0)$ \\
$x_{4}$ & $(7.4,7.8,8.4)$ & $(2.4,2.9,3.3)$ & $(8.3,8.8,9.3)$ & $(2.2,2.8,3.2)$ & $(2.2,2.7,3.2)$ & $(7.2,7.8,8.3)$ \\
\hline & & & & & & \\
\hline
\end{tabular}




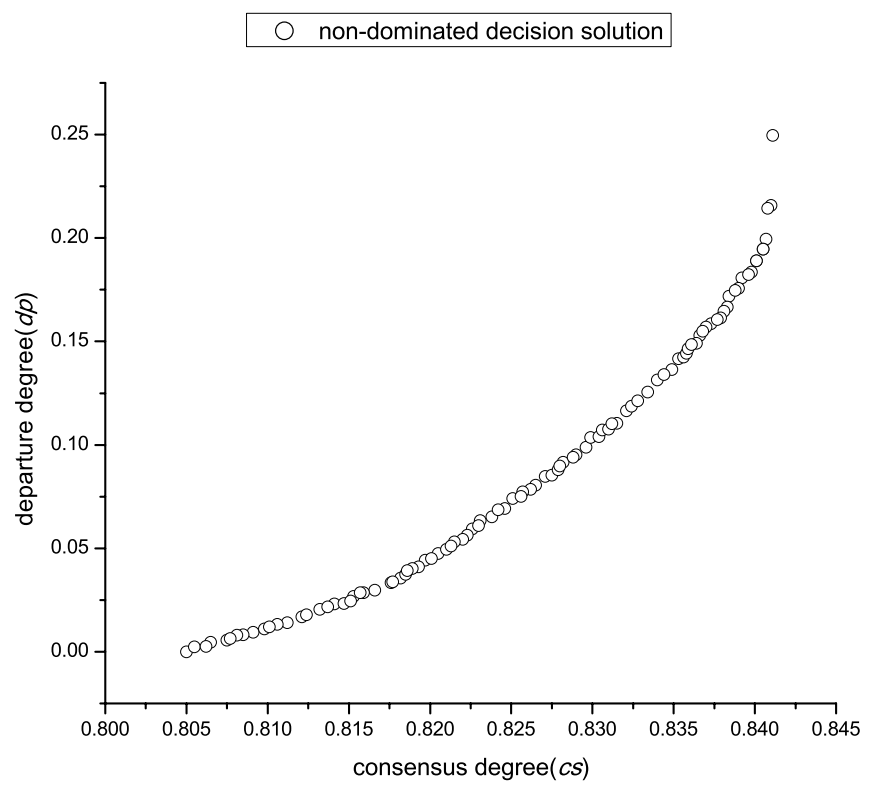

Figure 2: Obtained non-dominated decision solutions of the multi-objective optimization problem of initial decision preferences given by DMs.

Table 3: Decision matrix given by DM2 after the first round of interactive procedure

\begin{tabular}{ccccccc}
\hline & $c_{1}$ & $c_{2}$ & $c_{3}$ & $c_{4}$ & $c_{5}$ & $c_{6}$ \\
\hline$x_{1}$ & $(8.1,8.6,9.1)$ & $(1.4,2.2,2.7)$ & $(7.3,7.6,8.1)$ & $(2.1,2.7,3.2)$ & $(1.2,1.6,2.0)$ & $(8.0,8.4,8.9)$ \\
$x_{2}$ & $(7.3,7.6,8.2)$ & $(1.7,2.1,2.5)$ & $(8.3,8.8,9.2)$ & $(2.0,2.3,2.8)$ & $(1.6,1.9,2.4)$ & $(7.5,7.9,8.3)$ \\
$x_{3}$ & $(6.8,7.3,7.7)$ & $(1.1,1.5,2.3)$ & $(8.7,9.1,9.4)$ & $(1.4,2.2,2.7)$ & $(2.0,2.3,2.6)$ & $(7.2,7.8,8.3)$ \\
$x_{4}$ & $(7.7,8.2,8.9)$ & $(2.1,2.4,2.8)$ & $(7.6,8.2,8.9)$ & $(1.8,2.3,2.9)$ & $(1.5,1.8,2.3)$ & $(7.7,8.2,8.8)$ \\
\hline
\end{tabular}

Table 4: Decision matrix given by DM5 after the first round of interactive procedure

\begin{tabular}{ccccccc}
\hline & $c_{1}$ & $c_{2}$ & $c_{3}$ & $c_{4}$ & $c_{5}$ & $c_{6}$ \\
\hline$x_{1}$ & $(8.1,8.5,9.0)$ & $(1.4,1.8,2.4)$ & $(7.0,7.9,8.5)$ & $(1.4,1.8,2.4)$ & $(1.8,2.4,2.9)$ & $(7.3,7.8,8.3)$ \\
$x_{2}$ & $(6.8,7.2,8.0)$ & $(1.6,2.2,2.5)$ & $(7.8,8.3,8.9)$ & $(1.3,1.9,2.2)$ & $(1.3,1.8,2.2)$ & $(8.2,8.7,9.2)$ \\
$x_{3}$ & $(8.0,8.4,8.9)$ & $(1.5,1.9,2.4)$ & $(7.0,7.8,9.0)$ & $(1.7,1.9,2.4)$ & $(1.5,2.0,2.7)$ & $(8.5,8.9,9.2)$ \\
$x_{4}$ & $(7.4,8.0,8.4)$ & $(1.2,1.5,2.2)$ & $(8.4,9.0,9.4)$ & $(2.2,2.8,3.2)$ & $(1.7,2.3,2.8)$ & $(7.2,8.0,8.5)$ \\
\hline
\end{tabular}




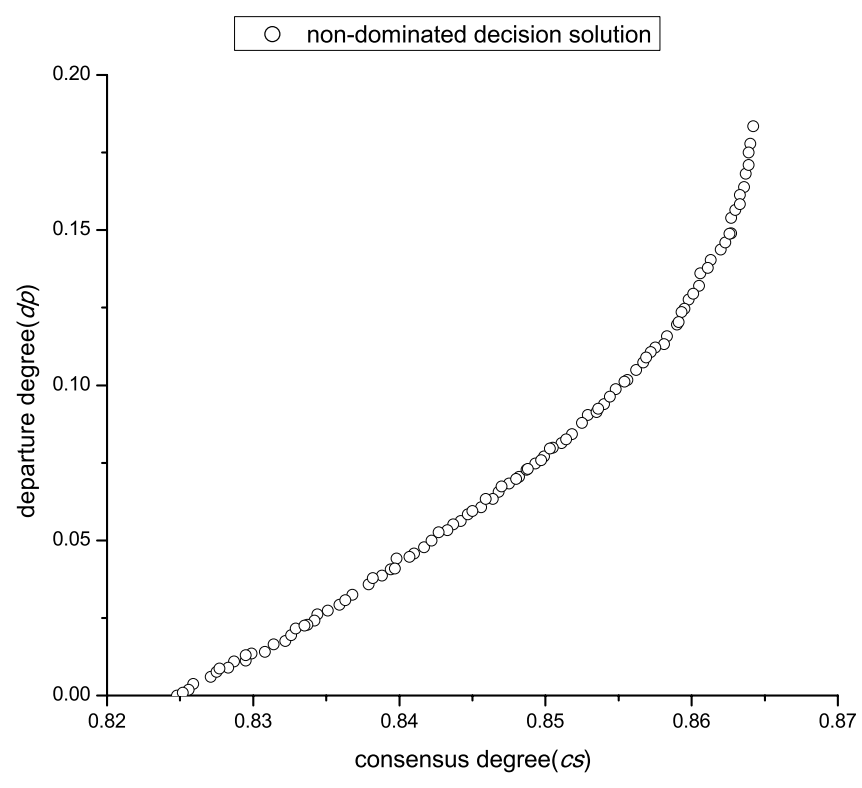

Figure 3: Obtained non-dominated decision solutions of the multi-objective optimization problem of the decision preferences given by DMs after the first round of interactive procedure.

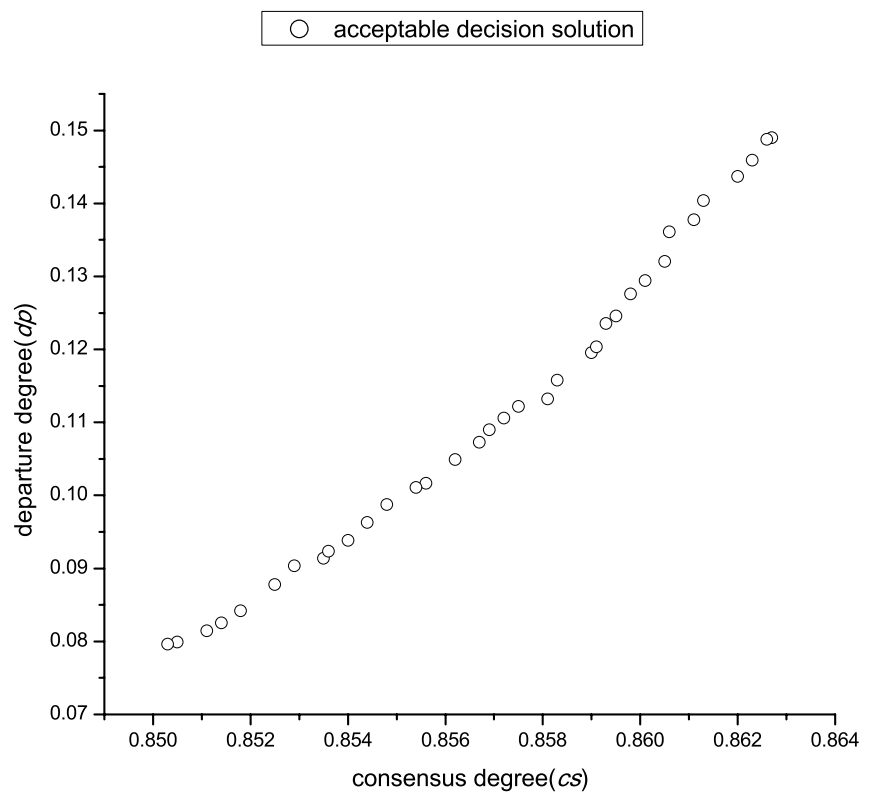

Figure 4: Obtained acceptable decision solutions of the multi-objective optimization problem of the decision preferences given by DMs after the first round of interactive procedure. 
Jian Xiong, Yingwu Chen, Kewei Yang et al.

Table 5: The transformed individual decision matrix of DM1-DM5 in the final decision solution

\begin{tabular}{|c|c|c|c|c|c|c|}
\hline DM1 & $c_{1}$ & $c_{2}$ & $c_{3}$ & $c_{4}$ & $c_{5}$ & $c_{6}$ \\
\hline$x_{1}$ & $(7.5,7.74,8.2)$ & $(7.5,8.10,8.5)$ & $(7.9,8.28,8.8)$ & $(7.1,7.42,7.8)$ & $(7.4,8.08,8.6)$ & $(8.3,8.82,9.3)$ \\
\hline$x_{2}$ & $(7.8,8.40,8.8)$ & $(7.7,8.14,8.7)$ & $(7.3,8.09,8.5)$ & $(7.3,7.78,8.2)$ & $(7.3,7.73,8.2)$ & $(8.3,8.90,9.4)$ \\
\hline$x_{3}$ & $(7.4,7.90,8.5)$ & $(8.0,8.50,8.8)$ & $(8.1,8.54,9.1)$ & $(7.5,8.27,8.7)$ & $(7.3,7.99,8.4)$ & $(7.4,8.01,8.9)$ \\
\hline$x_{4}$ & $(8.2,8.83,9.2)$ & $(7.0,7.70,8.2)$ & $(8.0,8.65,9.0)$ & $(6.9,7.34,7.9)$ & $(7.8,8.31,8.7)$ & $(7.9,8.43,9.0)$ \\
\hline \multicolumn{7}{|l|}{ DM2 } \\
\hline$x_{1}$ & $(8.1,8.67,9.1)$ & $(7.3,7.77,8.6)$ & $(7.3,7.75,8.1)$ & $(6.8,7.45,7.9)$ & $(8.0,8.49,8.8)$ & $(8.0,8.40,8.9)$ \\
\hline$x_{2}$ & $(7.3,7.60,8.2)$ & $(7.5,7.96,8.3)$ & $(8.3,8.70,9.2)$ & $(7.2,7.76,8.0)$ & $(7.6,8.10,8.4)$ & $(7.5,7.91,8.3)$ \\
\hline$x_{3}$ & $(6.8,7.38,7.7)$ & $(7.7,8.30,8.9)$ & $(8.7,9.18,9.4)$ & $(7.3,7.90,8.6)$ & $(7.4,7.72,8.0)$ & $(7.2,7.85,8.3)$ \\
\hline$x_{4}$ & $(7.7,8.23,8.9)$ & $(7.2,7.65,7.9)$ & $(7.6,8.32,8.9)$ & $(7.1,7.71,8.2)$ & $(7.7,8.21,8.5)$ & $(7.7,8.22,8.8)$ \\
\hline \multicolumn{7}{|l|}{ DM3 } \\
\hline$x_{1}$ & $(7.6,8.06,8.8)$ & $(7.1,7.59,8.0)$ & $(7.8,8.19,8.9)$ & $(7.2,7.66,7.9)$ & $(7.4,7.80,8.3)$ & $(8.2,8.51,9.0)$ \\
\hline$x_{2}$ & $(7.4,7.88,8.5)$ & $(7.6,8.12,8.7)$ & $(6.9,7.79,8.3)$ & $(7.8,8.30,8.7)$ & $(7.5,7.96,8.5)$ & $(8.6,9.00,9.4)$ \\
\hline$x_{3}$ & $(7.6,8.06,8.9)$ & $(7.4,7.94,8.2)$ & $(8.1,8.57,9.0)$ & $(7.2,7.74,8.2)$ & $(7.6,8.21,8.6)$ & $(8.0,8.50,8.9)$ \\
\hline$x_{4}$ & $(8.8,9.13,9.3)$ & $(7.0,7.51,8.1)$ & $(8.0,8.61,9.0)$ & $(8.0,8.44,8.9)$ & $(7.8,8.12,8.4)$ & $(7.8,8.31,8.8)$ \\
\hline \multicolumn{7}{|l|}{ DM4 } \\
\hline$x_{1}$ & $(8.0,8.43,8.9)$ & $(7.8,8.20,8.6)$ & $(7.7,8.22,8.6)$ & $(7.1,7.48,7.8)$ & $(7.6,8.34,8.6)$ & $(7.3,8.44,9.3)$ \\
\hline$x_{2}$ & $(7.8,8.25,8.8)$ & $(7.2,7.73,8.4)$ & $(7.0,7.65,8.1)$ & $(7.4,8.02,8.4)$ & $(7.1,7.55,8)$ & $(8.4,8.81,9.5)$ \\
\hline$x_{3}$ & $(7.6,8.25,8.6)$ & $(7.3,7.80,8.2)$ & $(8.1,8.78,9.1)$ & $(7.5,8.26,8.7)$ & $(7.3,7.85,8.4)$ & $(8.0,8.31,8.8)$ \\
\hline$x_{4}$ & $(8.0,8.52,9)$ & $(7.5,8.38,8.8)$ & $(8.4,8.97,9.3)$ & $(6.9,7.51,7.9)$ & $(7.8,8.57,8.7)$ & $(7.9,8.56,8.9)$ \\
\hline \multicolumn{7}{|l|}{ DM5 } \\
\hline$x_{1}$ & $(8.1,8.47,9)$ & $(7.6,8.21,8.6)$ & $(7.0,7.84,8.5)$ & $(7.6,8.20,8.6)$ & $(7.1,7.76,8.2)$ & $(7.3,7.88,8.3)$ \\
\hline$x_{2}$ & $(6.8,7.40,8)$ & $(7.5,7.85,8.4)$ & $(7.8,8.35,8.9)$ & $(7.8,8.11,8.7)$ & $(7.8,8.23,8.7)$ & $(8.2,8.70,9.2)$ \\
\hline$x_{3}$ & $(8.0,8.39,8.9)$ & $(7.6,8.20,8.5)$ & $(7.0,8.22,9.0)$ & $(7.6,8.09,8.3)$ & $(7.3,7.95,8.5)$ & $(8.5,8.90,9.2)$ \\
\hline$x_{4}$ & $(7.4,8.02,8.4)$ & $(7.8,8.49,8.8)$ & $(8.4,9.02,9.4)$ & $(6.8,7.53,7.8)$ & $(7.2,8.08,8.3)$ & $(7.2,8.08,8.5)$ \\
\hline
\end{tabular}


Table 6: The collective transformed individual decision matrix in the final decision solution

\begin{tabular}{cccccccc}
\hline & $c_{1}$ & $c_{2}$ & $c_{3}$ & $c_{4}$ & $c_{5}$ & $c_{6}$ \\
\hline$x_{1}$ & $(7.88,8.30,8.83)$ & $(7.47,7.97,8.45)$ & $(7.55,8.06,8.58)$ & $(7.15,7.64,7.99)$ & $(7.52,8.11,8.50)$ & $(7.79,8.40,8.97)$ \\
$x_{2}$ & $(7.43,7.91,8.47)$ & $(7.48,7.95,8.49)$ & $(7.43,8.08,8.57)$ & $(7.51,8.01,8.41)$ & $(7.44,7.90,8.35)$ & $(8.21,8.67,9.17)$ \\
$x_{3}$ & $(7.48,8.01,8.53)$ & $(7.56,8.11,8.48)$ & $(8.02,8.68,9.12)$ & $(7.41,8.05,8.50)$ & $(7.39,7.94,8.38)$ & $(7.84,8.32,8.81)$ \\
$x_{4}$ & $(8.04,8.55,8.97)$ & $(7.31,7.96,8.38)$ & $(8.09,8.72,9.13)$ & $(7.16,7.73,8.16)$ & $(7.67,8.27,8.52)$ & $(7.71,8.33,8.80)$ \\
\hline
\end{tabular}

the trade-off approach. As this process is out of the scope of our research, we assumed one of the acceptable decision solutions as the final one, which had a consensus degree $c s=0.8613$ and departure degree $d p=0.1404$. The transformed individual decision matrices of DMs are represented in Table 5. The collective transformed decision matrix is shown in Table 6 . With the collective decision matrix, we used the TOPSIS method presented by Yeh and Chang ${ }^{8}$ to calculate the preference value of each alternative and the results is shown in Table 7. Then, the ranking order of alternatives in the selected final decision solution is : $x_{4} \prec x_{1} \prec x_{3} \prec x_{2}$.

\section{Conclusion}

As the increasing complexity of the social-economic environment, many decision problems are no longer performed by a single DM, but involved a group of experts to achieve the decision solution. This paper focuses on MAGDM in which DMs express their absolute judgements on alternatives with respect to different evaluating attributes by fuzzy numbers. We employ consensus degree and departure degree to measure a decision solution from two different aspects. The former degree reflects the agreement level amongst all DMs and the latter degree is used to evaluate how far the DMs depart from their most preferable evaluation values. Based on these two conflicting measure degrees, the decision process of MAGDM is modelled as a multi-objective optimization problem. Then, a multi-objective evolutionary algorithm is employed to obtain the acceptable decision solutions. A decision support model (DSM) for MAGDM is proposed in this research. The proposed DSM is an iterative and interactive process which includes 5 implementing phases. With the proposed approach and model, we can avoid the situation that DMs severely depart from their preference while pursuing the maximum consensus degree of the decision solution, thus we can effectively obtain a more reliable decision result. For illustrating the feasibility of our approach, we take the alternative selection problem about a hydroelectric project as a case study and report the decision process and the decision results for the problem.

\section{Acknowledgments}

This research has been supported in part by the China Scholarship Council and National Natural Science Foundation of China under the Contrac$\mathrm{t}$ numbers 70971131, 70901074, 71001104 and 61271301 .

\section{References}

1. Utpal Bose, Anne M. Davey and David L Olson, "Multi-attribute utility methods in group decision making Past application and potential for inclusion in GDSS," Omega, 25, 691-706 (1997).

2. V. S. Lai, Bo K. Wong and W. Cheung, "Group decision making in a multiple criteria environment: A case using AHP in software selection," European Journal of Operational Research, 137, 134-144 (2002).

3. C. Kahraman, D. Ruan and I. Doğan, "Fuzzy group decision-making for facility location selection," Information Sciences, 157, 135-153 (2003).

4. C. E. Bozda , C. Kahraman and D. Ruan, "Fuzzy group decision making for selection among comput- 
Table 7: The preference value of each alternative in the final decision solution

\begin{tabular}{lcccc}
\hline Alternative & $x_{1}$ & $x_{2}$ & $x_{3}$ & $x_{4}$ \\
\hline Preference value & 0.6930 & 0.2270 & 0.4957 & 0.7308 \\
Ranking & 2 & 4 & 3 & 1 \\
\hline
\end{tabular}

er integrated manufacturing systems," Computers in Industry, 51, 13-29 (2003).

5. A. K. Choudhury, R. Shankar and M. K. Tiwari, "Consensus-based intelligent group decision-making model for the selection of advanced technology," Decision Support Systems, 42, 1776-1799 (2006).

6. J. Lu, G.Q. Zhang, D. Ruan, Intelligent multicriteria fuzzy group decision-making for situation assessments, Soft Comput 12, 289-299 (2008).

7. T. L. Saaty, Rank from comparisons and from ratings in the analytic hierarchy/network processes, European Journal of Operational Research 168, 557-570 (2006).

8. C.-H. Yeh, Y.-H. Chang, Modeling subjective evaluation for fuzzy group multicriteria decision making, European Journal of Operational Research 194, 464473 (2009).

9. Zeshui Xu, Multiple-attribute group decision making with different formats of preference information on attributes, IEEE Transaction on Systems, Man, and Cybernetics-Part B:Cybernetics 37, 15001511 (2007).

10. S.Saint and J.R.Lawson, "Rules for reaching cosensus: A modern approach to decision making," CA:Jossey-Bass, San Francisco, 1994.

11. J. Kacprzyk, M. Fedrizzi and H. Nurmi, Group decision making and consensus under fuzzy preferences and fuzzy majority, Fuzzy Set and Systems 49, 21-31 (1992).

12. E. Ephrati and J. S. Rosenschein, Deriving consensus in multiagent systems, Atificial Intelligence 87, 21-74 (1996).

13. F. Herrera, E. Herrera-Viedam and J. L. Verdegay, $A$ model of consensus in group decision making under linguistic assessments, Fuzzy Sets and Systems 79, 73-87 (1996).

14. F. Herrera, E. Herrera-Viedam and J. L. Verdegay, $A$ rational consensus model in group decision making under linguistic assessment, Fuzzy Sets and Systems 88, 31-49 (1997).

15. M. Fedrizzi,M. Fedrizzi and R. A. Marques Pereira, Soft consensus and network dynamics in group decision making, International Journal of Intelligent Systems 14, 63-77 (1999).

16. E. Herrera-Viedma, Modeling the retrieval process for an information retrieval system using an ordinal fuzzy linguistic approach, Journal of the American Society for Information Science and Technology 52, 460-475 (2001).

17. E. Herrera-Viedam and L. Martínez and F.Chiclana, A consensus support system model for group decisionmaking problems with multigranular linguistic preference relations, IEEE Transactions on Fuzzy Systems 13, 644-658 (2005).

18. P. Eklund, A. Rusinowska and H. de Swart, Consensus reaching in committees, European Journal of Operational Research 178, 185-193 (2007).

19. F.Mata,L.Martínez and E.Herrera-Viedam, An adaptive consensus support model for group decisionmaking problems in a multigranular fuzzy linguistic context, IEEE Transactions on Fuzzy Systems 17, 279-290 (2009).

20. G. Bordogna, M. Fedrizzi and G. Pasi, A linguistic modeling of consensus in group decision making based on OWA operators, IEEE Transactions on Systems,Man,Cybernetics-Part A 27, 126-132 (1997).

21. D. Ben-Arieh and Z. F. Chen, Linguistic-labels aggregation and consensus measure for autocratic decision making using group recommendations, IEEE Transactions on Systems,Man,Cybernetics-Part A 36, 558-568 (2006).

22. D. Ben-Arieh and T. Easton, Multi-criteria group consensus under linear cost opinion elasticity, Decision Support Systems 43, 713-721 (2007).

23. Zeshui Xu, An automatic approach to reaching consensus in multiple attribute group decision making, Computers \& Industrial Engineering 56, 1369-1374 (2009).

24. S. H. Kim and C. H. Han, An interactive procedure for multi-attribute group decision making with incomplete information, Computers \& Operations Research 26 (1999), 755-772.

25. Y. Y. Haimes, "Risk Modeling, Assessment, and Management," $2^{\text {nd }}$ edition, Wiley-Interscience, New York, 2004.

26. The Telegraph, "Amazon facing 'real-life Avatar' says James Cameron", 2010.

27. C.-H. Yeh, R. J. Willis and H.Deng et al, Task oriented weighting in multi-criteria analysis, European Journal of Operation Research 119, 130-146 (1999).

28. H.-J. Zimmermann, "Fuzzy set theory and its applications," $3^{\text {rd }}$ edition, Kluwer Academic Publishers, Boston, 1999. 
29. A. I. Olcer and A. Y. Odabasi, A new fuzzy multiple attribute group decision making methodology and its application to propulsion/manoeuvring system selection problem, European Journal of Operation Research 166, 93-114 (2005).

30. L. A. Zadeh, Fuzzy sets, Information and Control 8, 38-353 (1965)

31. T. L. Saaty, "The analytic hierarchy process," McGraw-Hill, New York, 1980.

32. N. Bryson, Group decision-makig and the analytic hierarchy process:Exploring the consensus-relevant information content, Computers and Operation Research 23, 27-35 (1996).

33. R. Zwich, E. Carlstein and D. V. Budescu, Measures of similarity among fuzzy concepts: A comparative analysis, International Journal of Approximate Reasoning 1, 221-242 (1987).

34. G. R. Klir and B. Yuan, "Fuzzy sets and fuzzy logic theory and applications," Prentice-Hall, Upper Saddle
River,NJ.

35. C.-T. Chen, Extensions of the TOPSIS for group decision-making under fuzzy environment, Fuzzy Sets and Systems 114, 1-9 (2000).

36. K. Deb, "Multi-Objective Optimization Using Evolutionary Algorithms," John Wiley \& Sons, New York, 2001.

37. K. Deb, A. Pratap, S. Agarwal, et al., A fast and elitist multiobjective genetic algorithm: NSGA-II, IEEE Transactions on Evolutionary Computation 2, 182197 (2002).

38. C. L. Hwang and K. Yoon "Multiple attribute decision making: Methods and application," Springer-Verlag, New York, 1981.

39. H. Deng, C.-H. Yeh and R. J. Willis, Inter-company comparison using modified TOPSIS with objective weights, Computers \& Operations Research 10, 645653 (2000). 